Case Reports

\title{
Purulent Infectious Myositis: Its Anguish and Ubiquitous!
}

\author{
Mohan, S., Mahendra Kumar Meena and Ravi Kant \\ Department of Internal Medicine, Aiims Rishikesh, India
}

\author{
Article history \\ Received: 10-08-2020 \\ Revised: 25-09-2020 \\ Accepted: 05-10-2020 \\ Corresponding Author: \\ Ravi Kant \\ Department of Internal \\ Medicine, Aiims Rishikesh, \\ India \\ Email: drkantr2006@gmail.com
}

\begin{abstract}
Purulent Infection Myositis (PIM) which was formerly called as tropical myositis is an acute or sub-acute primary infection of striated muscles. It affects all age groups, both sexes and occurs in tropical as well as temperate climates. Most frequently encountered culprits are skin commensals like staphylococci aureus. It is predominantly reported in immunocompromised individuals and with pre-existing muscle abnormalities like strain, trauma, parasite or another inciting event. Gramnegative bacilli myositis in an immunocompetent individual is rare. We report a case of Escherichia coli PIM in an immunocompetent patient with no risk factors and muscle abnormality. This helps early consideration of proper antibiotic therapy in an immunocompetent patient with pyomyositis and helps avoid complications associated with pyomyositis.
\end{abstract}

Keywords: Tropical Myositis, Gram Negative Bacteria, Immunocompetent, Escherichia coli

\section{Background}

Purulent Infectious Myositis (PIM) is a subacute purulent infection of the skeletal muscles (Crum-Cianflone, 2008). The first reported case of PIM was reported in 1971 (Levin et al., 1971). Previously it was referred to as 'Tropical myositis' considering most of the reported cases were from tropical countries (Habeych et al., 2020). Now it's considered a ubiquitous disease affecting people in temperate as well as tropical climate. Hence the terminology has been changed to purulent infectious myositis (Shepherd, 1983). It affects all age groups, both sexes and both immunocompromised and immunocompetent individuals (Habeych et al., 2020). Extensive PIM is most commonly affects the immunocompromised individual with staphylococcus aureus as the most common etiologic agent identified (Habeych et al., 2020). PIM is associated with significant morbidity and mortality due to complications like Meningitis, septic emboli, sepsis, compartment syndrome, septic arthritis, pancarditis and multi-organ dysfunction (Al-Najar et al., 2010).

\section{Case Presentation}

A 34 years lady with no previous known comorbidities presented to our institute with complaints of pain in all limbs for 7 days duration following development of furuncle over left buttock 12 days back for which she didn't take any medications. Three days into illness she developed high grade fever, pain and swelling of left thigh associated with difficulty in walking. Her menstrual history was normal and her obstetric history is 12 years of married life with 2 children both were full-term normal vaginal delivery. There was no history of any substance abuse. With these complaints' patient visited a nearby hospital where her blood investigations done which showed high Creatinine Phosphokinase (CPK) values of 6000 units/dl. MRI of $\mathrm{b} / \mathrm{l}$ thigh was done which showed swelling/oedema/inflammation involving the pelvic, gluteal and bilateral thigh muscles appearing hypointense on $\mathrm{T} 1$ and hyperintense on $\mathrm{T} 2 \mathrm{w}$ images with no obvious collection or mass consistent with inflammatory myositis (Fig. 4). During this period her symptoms worsened with development of severe pain, swelling and redness in all four limbs (proximal $>$ distal) (Fig. 1). She was referred to our centre with a provisional diagnosis of inflammatory myositis. On presentation she was bedridden with severe pain in minimal of activities.

On examination, she was conscious oriented, febrile with a temperature of $39.3 \mathrm{C}$ and other vitals were normal. She had pain in all limbs on both active and passive movements. Erythema was present on medial side of bilateral upper limb and thighs which were warm and tender on light palpation. Power could not be assessed due to pain on all limb movements. All other systemic examination was within normal limits. 


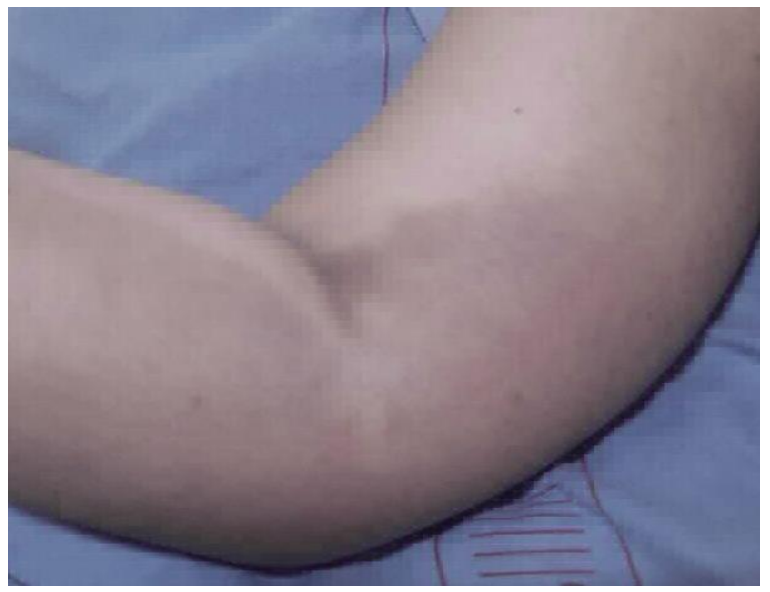

Fig. 1: Erythematous indurated area in the medial side of the right arm and forearm suggestive of pus collection

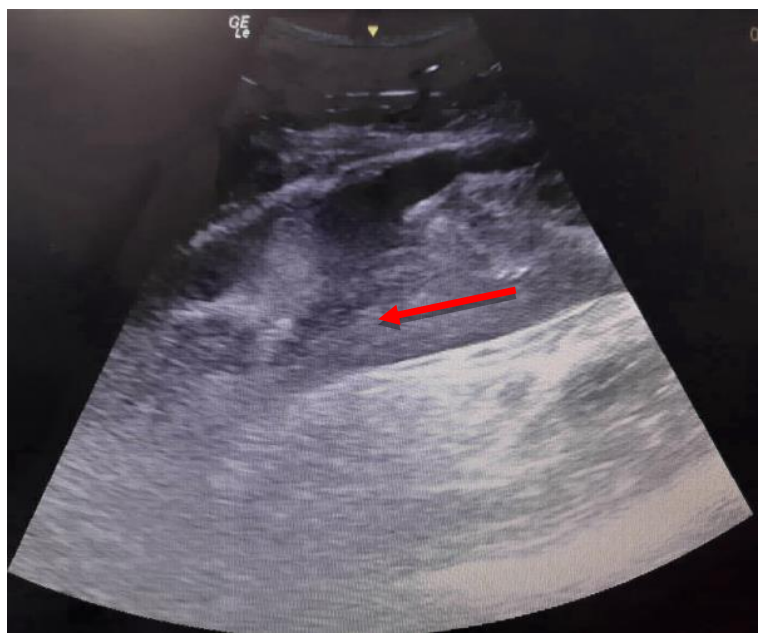

Fig. 2: A large pocket of the collection with moving internal echoes is seen in the intermuscular plane of the left thigh

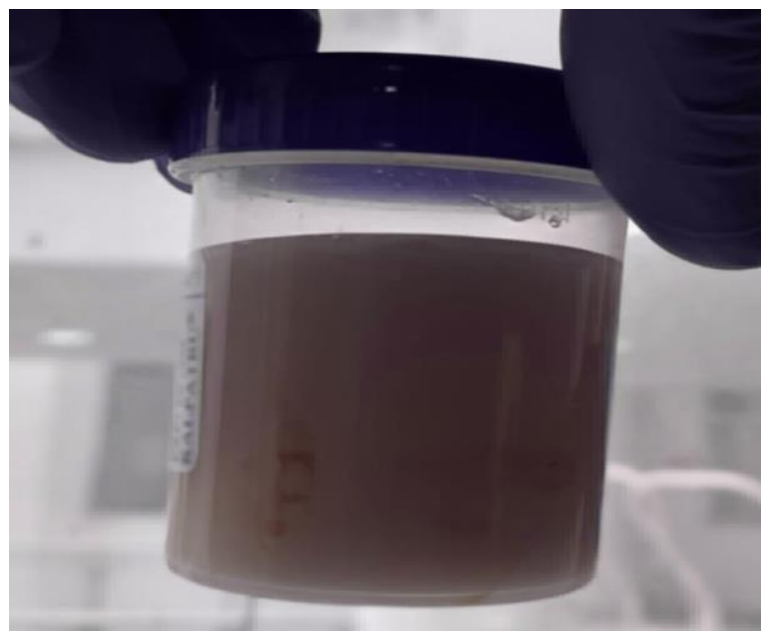

Fig. 3: Pus aspirated form the site

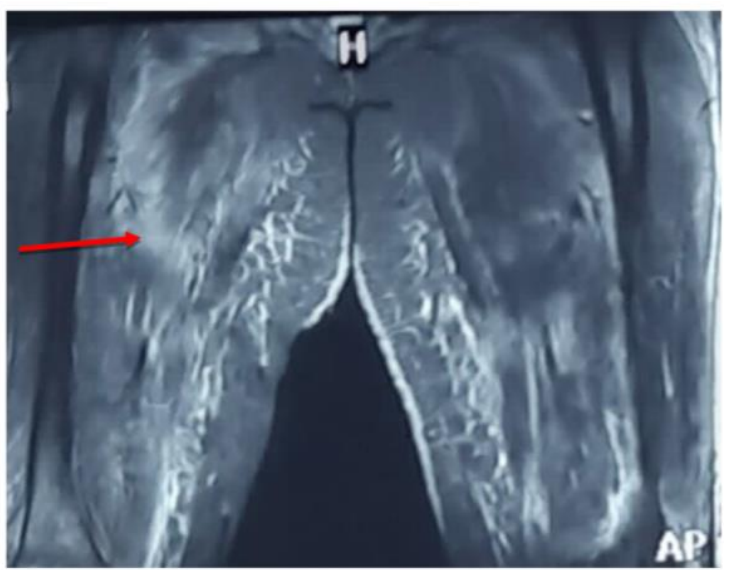

Fig. 4: Magnetic Resonance Imaging (MRI)-Diffuse swelling/ oedema/inflammation seen involving the pelvic, gluteal and bilateral thigh muscles appearing hyperintense on $\mathrm{T} 2 \mathrm{w}$ images with no obvious collection or mass

\section{Investigations if Relevant}

Her Total leucocyte count $(14,450$ cells/ml), C-reactive protein $(694 \mathrm{mg} / \mathrm{dl})$ and Erythrocyte sedimentation rate (ESR-117 mm/h) were elevated. Procalcitonin was also elevated $(32.5 \mathrm{ng} / \mathrm{dl})$ and a provisional diagnosis of tropical pyomyositis was considered. Bedside USG (Fig. 2) showed pus (Fig. 3) collection in intermuscular planes in b/l upper and lower limbs. Aspiration revealed thick, turbid, yellow coloured pus which was sent for culture. Transthoracic echo was done to rule out infective endocarditis which was normal. Her electromyography study was done which showed features of inflammatory myopathy (increased spontaneous activity, insertional activity and Polymorphic small-amplitude Motor unit action potential with increased recruitment). Her immunoglobulin profile (IgG, IgM, IgA and $\operatorname{IgE}$ ) as sent to rule out immunodeficiency which came to normal. Her autoimmune myositis panel $(\mathrm{Ku}, \mathrm{Mi}-2$ beta, PM-Sc100, PM-Sc75, JO-1, SRP, PL-7, PL-12, EJ, OJ and RO-52) was negative. Her CPK (4324 IU/mL) was elevated. Her blood culture was sterile while pus culture revealed Escherichia Coli sensitive to amikacin. She was advised for magnetic resonance imaging pelvic region and bilateral lower limb to look for the extent of a collection in lower limbs which showed multiple peripherally enhancing intercommunicating intramuscular intercommunicating intramuscular collection in bilateral thigh involving all three compartments with largest in medial compartment of left thigh $(2.2 \times 8.9 \times 10 \mathrm{~cm})$ are also seen in left thigh (Fig. 5). Her fever subsided with treatment but swelling persisted despite repeated drainage. Pigtail insertion in the left thigh was done and rest of the sites were drained under USG guidance. Her Total leucocyte and count, CPK NAC and other inflammatory markers are normalized over next 3 weeks and she was discharged in stable condition. 


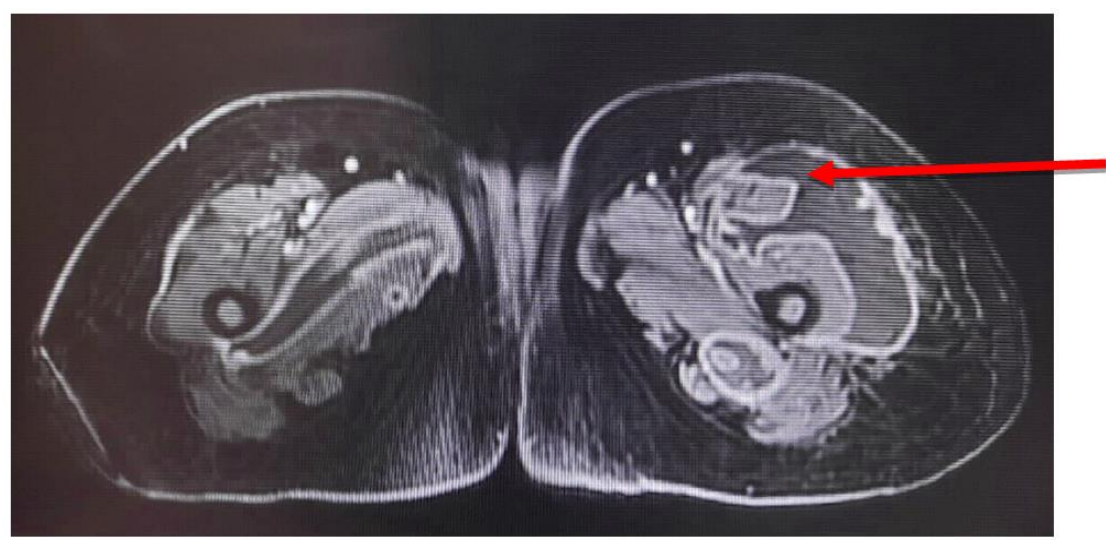

Fig. 5: MRI - the large collection measures- $2.2 \times 8.9 \times 10 \mathrm{~cm}(\mathrm{AP} X \mathrm{TR} \times \mathrm{CC})$ in medial compartment of right thigh.

\section{Treatment}

Patient was started on a broad-spectrum antibiotic (piperacillin-tazobactam) initially and later antibiotics were changed to Amikacin according to sensitivity. She was continued on same antibiotic for 21 days. She was advised for protein diet and regular physiotherapy. Pigtail insertion in the left thigh was done and rest of the sites were drained under ulatrasound guidance.

\section{Out Come and Follow-up}

Her Total leucocyte and count, CPK NAC and other inflammatory markers normalized over next 3 weeks and pigtail removal was done. She was discharged in stable condition.

\section{Discussion}

Purulent infection myositis is formation of pus in intermuscular planes most commonly encountered during bacteraemia by gram positive organisms. There are multiple hypotheses proposed for the pathogenesis of PIM. Trauma leading to invasion by pathogenic organism was the most prevalent hypothesis initially as most of PIM cases were young males and muscle affected in these patients were those used to get injured during daily work (Diamandakis and Grose, 1994; Theodorou et al., 2007). The next hypothesis of PIM was due to vitamin deficiency (thiamine) (Engel, 1981) and parasites (Toxocara canis, trichinella, Dracunculus medinesis) (Habeych et al., 2020) with concurrent bacteremia. Presently PIM is considered to occur as a complication of transient bacteraemia in patients with pre-existing muscle abnormality (strain, trauma, parasite, or another inciting event) (Domínguez-Pinilla et al., 2015). Evolutionarily iron sequestration in intracellular spaces act as defence mechanism against bacterial proliferation. Muscle damage releases free iron from myoglobin providing adequate environment for bacterial proliferation and predisposing patients to PIM. Viral infection, hepatitis B carrier state, IV drug abuse, malnutrition, viral infection, Immunodeficiency and malignancy are the common predisposing conditions for pyomyositis (Habeych et al., 2020). Our patient did not have any comorbidities and extensive workup did not reveal any immunodeficiency state.

The most common etiologic agent for PIM is staphylococcus aureus followed by streptococci pyogens and less frequently Escherichia Coli (Sharma et al., 2011). E. coli is most commonly associated with urinary tract infection frequently identified in the skin around perineal region (Foxman, 2002). Multiple risk factors are typically common with the extraintestinal manifestation of E. coli (Russo and Johnson, 2003). PIM is a rare extraintestinal manifestation of $E$. coli (Cooke et al., 2010). E. coli PIM is typically most common with immunocompromised individuals and it is rare in immunocompetent individuals (Vigil et al., 2010). In our patient furuncle over the buttock region may have led to bacteraemia with seeding of different muscles ultimately leading to pyomyositis.

PIM divided into three clinical stages, stage I: Invasive stage characterized by bacterial invasion of muscle manifesting as fever associated with muscle pain, stage II: Purulent or suppurative stage characterized by purulent collection. Most of the patients present to health care in the second stage of the disease. Stage III is the late-stage characterized by complications of myositis like compartment syndrome, infective endocarditis, sepsis, multi organ dysfunction (Habeych et al., 2020). Our patient also presented to us in stage II that is the purulent stage.

In the management of PIM, gram-negative coverage is generally advocated for patients with multiple comorbidities and immunocompromised states (Habeych et al., 2020). Our case highlights the need of early gram-negative antibiotic therapy in PIM to avoid complications and reduce morbidity and mortality. 
PIM is a great masquerade, a wide range of differential diagnoses and lack of early specific signs (Shepherd, 1983). Extensive pyomyositis must be differentiated from polymyositis (Chauhan et al., 2004). In our case, initial differentiation was difficult because the patient is a middle-aged female presented with extensive myositis without any comorbidities. She was diagnosed as PIM based on pus culture report and MRI findings.

\section{Learning Points/Take Home Messages 3-5 Bullet Points}

1. Extensive PIM can occur in an immunocompetent individual by gram-negative bacteria.

2. Early aggressive antibiotics therapy should be instituted to avoid complications and gram-negative cover should be considered

3. PIM can mimic as polymyositis in the early stages

4. Its associated with significant morbidity to patient with prolonged hospital stay with severe pain and disability

\section{Acknowledgment}

The authors would like to acknowledge all India Institute of Medical Sciences, Rishikesh.

\section{Author's Contributions}

Mohan. S.: Manuscript preparation, data collection, study design.

Mahendra Kumar Meena: Manuscript preparation, Manuscript editing.

Ravi Kant: Manuscript review, guidance, corresponding author.

\section{Ethics}

This article is original and contains unpublished material. The corresponding author confirms that all of the other authors have read and approved the manuscript and no ethical issues involved.

\section{References}

Al-Najar, M., Obeidat, F., Ajlouni, J., Mithqal, A., \& Hadidy, A. (2010). Primary extensive pyomyositis in an immunocompetent patient: case report and literature review. Clinical rheumatology, 29(12), 1469-1472.

Chauhan, S., Jain, S., Varma, S., \& Chauhan, S. S. (2004). Tropical pyomyositis (myositis tropicans): current perspective. Postgraduate Medical Journal, 80(943), 267-270.
Cooke, N. M., Smith, S. G., Kelleher, M., \& Rogers, T. R. (2010). Major differences exist in frequencies of virulence factors and multidrug resistance between community and nosocomial Escherichia coli bloodstream isolates. Journal of clinical microbiology, 48(4), 1099-1104.

Crum-Cianflone, N. F. (2008). Bacterial, fungal, parasitic and viral myositis. Clinical Microbiology Reviews, 21(3), 473-494.

Diamandakis, V., \& Grose, C. (1994). Bad consequences of bicycle accidents: Pyomyositis. Pediatric infectious disease journal, 13(5), 422-425.

Domínguez-Pinilla, N., Villora-Morcillo, N., Caro-Barri, A., Vivanco-Martinez, J., \& Gonzalez-Granado, L. I. (2015). Pyomyositis and bacteremia due to Staphylococcus aureus as onset of acute promyelocytic leukemia. Pediatrics International, 57(5), 1053-1054.

Engel, D. (1981). Tropical pyomyositis, a thiaminedeficiency disease. Medical hypotheses, 7(3), 345-352.

Foxman, B. (2002). Epidemiology of urinary tract infections: incidence, morbidity and economic costs. The American journal of medicine, 113(1), 5-13.

Habeych, M. E., Trinh, T., \& Crum-Cianflone, N. F. (2020). Purulent infectious myositis (formerly tropical pyomyositis). Journal of the Neurological Sciences, 116767.

Levin, M. J., Gardner, P., \& Waldvogel, F. A. (1971). Tropical pyomyositis: an unusual infection due to Staphylococcus aureus. New England Journal of Medicine, 284(4), 196-198.

Russo, T. A., \& Johnson, J. R. (2003). Medical and economic impact of extraintestinal infections due to Escherichia coli: focus on an increasingly important endemic problem. Microbes and infection, 5(5), 449-456.

Sharma, U., Schwan, W. R., \& Agger, W. A. (2011). Escherichia coli pyomyositis in an immunocompromised host. WMJ: official publication of the State Medical Society of Wisconsin, 110(4), 182.

Shepherd, J. J. (1983). Tropical myositis: is it an entity and what is its cause?. The Lancet, 322(8361), 1240-1242.

Theodorou, S. J., Theodorou, D. J., \& Resnick, D. (2007). MR imaging findings of pyogenic bacterial myositis (pyomyositis) in patients with local muscle trauma: illustrative cases. Emergency Radiology, 14(2), 89-96.

Vigil, K. J., Johnson, J. R., Johnston, B. D., Kontoyiannis, D. P., Mulanovich, V. E., Raad, I. I., ... \& Adachi, J. A. (2010). Escherichia coli pyomyositis: an emerging infectious disease among patients with hematologic malignancies. Clinical infectious diseases, 50(3), 374-380. 